\title{
Cesare Rinaldi, Guido Reni e l'ecfrasi del moderno
}

\section{Giorgio Forni}

Università di Messina

giorgio.forni@unime.it

\section{Riassunto}

Affinché la descrizione di opere d'arte potesse trovare spazio nell'ambito della lirica moderna era necessario andare oltre il primato classicistico della poesia sulla pittura e riconoscere un'affinità e un'intesa paritaria fra arti sorelle. Con le Rime nuove del 1603 il poeta Cesare Rinaldi, amico dei Carracci e di Guido Reni, inaugura una nuova possibilità di dialogo tra pittura e poesia su base lirica e soggettiva, ponendo al centro di quel canzoniere il mito luminoso di Barbara come immagine del sentimento polemicamente al di là del "vero" e del "falso".

Parole chiave: Cesare Rinaldi; Torquato Tasso; Guido Reni; i Carracci; Barocco; ecfrasi lirica; arti sorelle; individualità moderna.

\section{Abstract. Cesare Rinaldi, Guido Reni and the Ecphrasis of the Modern}

In order for the description of works of art to find a place in modern lyric poetry, it was necessary to go beyond the classicist primacy of poetry over painting and recognise an affinity and mutual exchange between sister arts. With his Rime nuove of 1603, the poet Cesare Rinaldi, a friend of the Carraccis and Guido Reni, inaugurated a new possibility of dialogue between painting and poetry on a lyric and subjective basis, placing at the centre of that book of rhymes the luminous myth of Barbara as an image of sentiment polemically beyond "true" and "false".

Keywords: Cesare Rinaldi; Torquato Tasso; Guido Reni; the Carraccis; Baroque; Lyric Ekphrasis; Sister Arts; Modern Individuality. 
"Ainsi le meilleur compte rendu d'un tableau pourra être un sonnet ou une élégie" Charles Baudelaire, Salon de 1846. I. À quoi bon la critique?

\section{1. "Una vilissima e Barbara carogna"}

Nel suo elogio funebre All'illustrissimo signor marchese Virgilio Malvezzi per la morte del signor Cesare Rinaldi, Gioan Pellegrino Pancaldi ricorda che quel "Sole" di poesia "per maggior spazio di tempo vagheggiò le allettatrici delizie di questo mondo" e spiega argutamente la sua morte col fatto che "era tanto liberale de' suoi affetti che non ricusò di cederli alla più odiosa cosa del mondo" (Pancaldi, 1636, pp. 6-7). Fino all'ultimo sembra gravare sul poeta un'ombra, un vago sospetto di debolezza morale che vela per un attimo il fulgido ritratto commemorativo del Rinaldi come gentiluomo e maestro di cultura valente e amabile. Qualcosa di simile traspare anche nel sonetto introduttivo Vinse barbare genti, ed a l'Impero con cui il conte Ridolfo Campeggi raccomandava nel 1619 la raccolta emendata e selettiva delle sue Rime celebrando il ritrovato dominio sopra le passioni del "pio" Cesare ormai "d'ogni ammenda scarco". ${ }^{1}$ Ma per capire questa punta di rigore moralistico che si mescola agli elogi per l'anziano poeta occorre fare qualche passo indietro.

Di nobile famiglia bolognese, il Rinaldi aveva rinunciato da giovane ai possedimenti ereditari per un "onesto assegno" che gli permettesse di dedicarsi alle lettere in piena libertà e di condurre la vita a suo modo. Ma era proprio negli anni di quella giovinezza fervida e curiosa che egli aveva cominciato a seguire una "cieca voglia in umiltà superba", una "colpa", un tenace "errore", al punto da trovarsi pubblicamente escluso dal principale sodalizio letterario della Bologna del tempo, l'Accademia dei Gelati, raccolta intorno ai valori del decoro aristocratico e di un moderno idealismo platonizzante. Lo attesta fra l'altro un sonetto incluso nelle sue Rime del 1591, Gelati spirti, il vostro gielo è fiamma: "Al vostro alto valor picciola dramma / giunger vorrei, ch'umile il cor vi serba; / ma, cieca voglia in umiltà superba!, / non segue lento bue veloce damma. / Or se nel vostro Sol non vuol ch'io splenda / mia colpa, almen dal folgorar di Giove / l'ombra del vostro lauro mi difenda. / Deh gradite il desire, amor lo move; / deh scusate l'error, tempo l'emenda; / ma il giel, ch'arde il mio cor, nulla rimove". ${ }^{2}$ Ora, per intendere l'ingegnoso paradosso con cui

1. Rinaldi, 1619, p. 9. È un volume che propone un'ampia scelta delle rime già edite come dichiara anche il motto sul frontespizio DELECTA DILECTIS e l'avvertenza $A$ ' lettori: "Tanto è svogliato di poesie l'autore delle presenti rime, che quand'io dissi di ristamparle, egli apertamente negommi ogni aiuto e di rifforma e di correzzione; alla fine mi compiacque, con patto ch'io dovessi rinovar quella protesta fatta da lui nelle passate impressioni; che, quanto egli ha detto dell'amor terreno, esaltando l'umana bellezza con epiteti sovraumani, tutto è stato uso di poetico stile, e non difetto di volontà corrotta" (p. 8). Cfr. al riguardo Rinaldi, 1620, pp. 169-170.

2. Rinaldi, 1591, p. 158. Era servito a poco alternare il repertorio voluttuoso del madrigale con la meditazione morale del sonetto nella Parte terza del 1590. Sull'esclusione dall'Accademia 
il Rinaldi chiude il sonetto, non va dimenticato che l'impresa collettiva dei Gelati raffigurava una selva di alberi coperti di neve col motto "Nec longum tempus": per essi il gelo della materia e dei sensi si sarebbe presto disciolto al tepore dell'eterna primavera delle idee, mentre la "cieca voglia", il "gelo" che "arde" il cuore del poeta non poteva essere estinto o superato. Ancora nel 1607, felicitandosi con il Campeggi per il suo Filarmindo, il Rinaldi riconosceva la propria indegnità di poeta sviato e spregevole: "Sì potess'io lodarti, alma gentile, / sì non fosse il mio carme un cieco inferno, / un fantasma d'error notturno e vile! / Ma non hanno in tuo pro florido stile / i GeLATI? i SelvagGr? Ah, che 'l mio Verno / a ragion non può star col loro Aprile" (Campeggi, 1607, c. A3r). Ed è un "Verno" di ombre terrene che si contrappone a un "Aprile" luminoso e ormai precluso, a un "florido stile" educato dal platonismo. Di lì a pochi anni il Rinaldi avrebbe rinnegato il proprio ostinato errore assumendo il "fermo proponimento di non più attender alla Poesia" e facendo anzi solenne "giuramento d'abbandonar le Muse". Nelle sue tarde lettere egli continua a scusarsi e giustificarsi attribuendo alla passione per la poesia l'origine delle proprie colpe: "io $[. .$.$] sempre ferneticai dietro a poesie vane e fra tante vanità$ poco mancò ch'io non divenissi superbo: ora me ne pento", scrive il 14 maggio 1611; "in somma è per me finita la Poesia, così non foss'ella mai cominciata", ribadisce il 2 settembre 1613; "pur troppo la poesia m'ha rovinato, e non fa per me l'accettar di nuovo i miei nemici in casa", dichiara ancora l'1 dicembre $1618 .^{3}$ Ed è un combattimento interiore contro le lusinghe della poesia che avrà poi tanti altri esempi nel primo Seicento. ${ }^{4}$

Chi si chieda quale sia l'errore di cui il Rinaldi non cessa di pentirsi, potrebbe forse trovare una prima risposta in un singolare episodio biografico. Ciò di cui egli si pente pare sia anzitutto l'amore per Barbara e la volontà di rendere pubblica la relazione del nobile e affermato poeta quarantenne con quella giovane prostituta poco più che diciottenne. ${ }^{5}$ Un manoscritto della Biblioteca dell'Árchiginnasio e uno della Biblioteca Universitaria di Bologna conservano infatti una lettera e un capitolo satirico anonimi intorno a quella vicenda scandalosa che dovette mettere a rumore la buona società bolognese

dei Gelati si veda Besomi, 1969, pp. 101-107, e Ritrovato, 2005, pp. 30-31, 98 e passim.

3. Cfr. Rinaldi, 1617, pp. 232, 239, 244, 147, e Rinaldi, 1620, p. 223. Una rassegna dei numerosi brani delle Lettere in cui il Rinaldi esprime rammarico e pentimento per la propria esperienza di poeta si legge in Ritrovato, 2005, pp. 98-101.

4. Cfr. Forni, 2018, pp. 33-53 e 159-180. Non si tratta però di una rinuncia penitenziale all'arte in genere: per il Rinaldi, come risulta dalle Lettere, abbandonare le Muse e rifiutare la poesia non significava non occuparsi più di pittura, musica e teatro. Quel pentimento riguardava solo la particolare forma di poesia che egli aveva scelto.

5. Cfr. Rinaldi, 1603, p. 69: "Di nove mesi il diciottesim'anno / passato, [...] / Morte, turbo crudel, svelse le rose, / squarciò le reti, impallidì gli Amori [...]". Poco altro si ricava dalle Rime nuove: Barbara chiede al poeta di cantare l'amica Flaminia (XLI); le lodi poetiche di Barbara vengono biasimate da donne perfide e villane (XLVI-XLVII); il poeta manda a Barbara una gazza in dono (LI-LII); Barbara veste di nero come se fosse a lutto (LIV-LV); durante l'inverno cade malata (LX-LXI) e muore (LXII); il poeta invita a celebrarne la tomba (LXIII-LXIX). 
se ancora quindici anni dopo il Campeggi vi poteva alludere nel sonetto Vinse barbare genti. Ecco la lettera, di poco anteriore al 1603:

Lettera di un nobile signore scritta ad altro suo amico, con la quale pretende riprendere la pazzia con cui il Rinaldi, celebre poeta di questa città di Bologna, ha intrapreso di poeticamente lodare le bellezze d'una pubblica meretrice nomata Barbara.

Ill[ustrissi]mo Sig[no]re,

il Rinaldi, poeta d'assai celebre grido, spendendo malamente il virtuoso talento che dal Cielo ha prodigiosamente avuto nelle lodi d'una vilissima e Barbara carogna, è stato a' dì passati in forma di fraterna correzione ripreso da me col capitolo qui congiunto. Egli fatto incorregibile va anco perseverando nella sua sciocchezza, et io alla seconda riprensione ho voluto chiamar V.S. Ill[ustrissi]ma, et altri di sua nobile conversazione, accioché come amici di questo miserello, mi servano per testimonii degli amorevoli racordi, che io li porgo, intendendo io (quando egli non cangi pensiero) di fargliene poi pubblica querela d'avanti a tutto il Collegio degli Dei, ma in particolare al Tribunale di Apollo, et delle sue sacrate Muse, acciò si neghi per l'avvenire l'addito di Parnaso ad un celebratore di soggetti infami. Aiutilo V.S. Ill[ustrissi]ma con amorevoli ammonizioni, che n'ha bisogno, et il Cielo la feliciti. ${ }^{6}$

Se poi si dà credito al capitolo satirico, si direbbe che il Rinaldi abbia avuto per qualche tempo l'intenzione di ufficializzare quel rapporto in nome di una piena sincerità sentimentale e poetica che rifiuta l'ipocrisia di "amar di contrabando" e l'espediente di cantare "qualche nobil soggetto alla Platonica" per poi "sfogare" sottobanco gli appetiti erotici "con le pubbliche donne da diletto" senza rischiare di "andarvi in bordell'a inamorare". ${ }^{7}$ Più che una "fraterna correzione" fatta di "amorevoli racordi", quella dell'anonimo era una

6. Cfr. Bologna, Biblioteca Universitaria, ms. 683, cc. $150 r-152 v$, e Biblioteca dell'Archiginnasio, ms. B. 3635.3, pp. 37-40. Anche nel capitolo satirico Or si Rinaldi, che la Musa vostra Barbara è additata come "puttana", "carogna" e "infame bagascia di bordello" a cui il Rinaldi vorrebbe legarsi con "infame laccio". Un madrigale scherzoso sul "venale Amor" era già in Rinaldi, 1590, p. 48: "venale Amor d'amor merca il tesoro, / dona l'Or, toglie l'or, cambia Oro in oro".

7. Cfr. Or si Rinaldi, che la Musa vostra, vv. 49-78: "Io la protezion vostra pigliando, / gli ho ben detto, tra molte altre cosette, / che non volete amar di contrabando, / e però volentier siete alle strette / venuto con sì vaga damigella / come cosa daziata alle Bollette. / Ma se ben la ragione è buona e bella, / il Mondo, accettar mai non la volendo, / me l'ha fatta tornar nella scarsella. / Or perché volentieri vi difendo / dalle lingue pestifere di drago, / per andar meglio il vostro error coprendo, / gli ho replicato che voi fate il vago / con le pubbliche donne da diletto / tolte dal Torlione o 'l Frassinago, / perché queste signore da rispetto / son nei giuochi d'amor sì male avvezze, / che più si muove il capezzal del letto. / Onde se non san far quattro carezze / avete ben ragione d'incacarne / a lor nobilitade, a le bellezze. / Che quando s'è tentato dalla carne, / d'una madonna onesta o d'una monica / non s'ha bisogno, e non si sa che farne. / Ma un uom, ch'avea sul piè longa la tonica, / m'ha risposto che voi dovreste amare / qualche nobil soggetto alla Platonica, / e che potreste ben talor sfogare / questo vostro appetito carnalaccio / senz'andarvi in bordell'a inamorare". Sull'inclinazione alla "sincerità" del Rinaldi insisterà poi Brusoni, 1647, p. 102: "era il Rinaldi d'animo così candido ed ingenuo che non avrebbe tacciuto la verità de' suoi sentimenti per tutto l'oro del mondo". 
denuncia in versi conclusa dall'auspicio non troppo scherzoso di vedere il poeta "incatenato" in un "Ospedale de' pazzi". A quell' attacco il Rinaldi rispose con la corona di sonetti contro gli "oltraggi" di un "tonante Momo" che si legge in apertura delle Rime nuove del 1603 (I-XVI). Non era solo un gioco letterario, e basta dare ascolto al sonetto proemiale per cogliere il senso polemico e difensivo di quella raccolta:

\begin{tabular}{|c|c|}
\hline $\begin{array}{l}\text { Or si Rinaldi, che la Musa vostra } \\
\text { vv. } 43-48\end{array}$ & $\begin{array}{l}\text { Rime nuove I } \\
\text { vv. } 9-14\end{array}$ \\
\hline $\begin{array}{l}\text { Onde parmi veder, che in questo caso } \\
\text { da tutte le persone letterate } \\
\text { vi si cominci molto a dar del naso. } \\
\text { In somma spiace a tutte le brigate } \\
\text { che voi questa puttana celebrando } \\
\text { di voi medesmo non vi vergogniate. }\end{array}$ & $\begin{array}{l}\text { O Lincei nel mio amor, poco vedete, } \\
\text { ché quanto di lei parlo è un'ombra, un sogno, } \\
\text { ma più del cieco Amor ciechi voi sete. } \\
\text { Sol nel mio stil barbaro nome agogno, } \\
\text { perch'ei l'ingemma, e d'altro i' non ho sete, } \\
\text { né meco di me stesso mi vergogno. }\end{array}$ \\
\hline
\end{tabular}

Così, a difesa della propria poesia e forse anche della propria libertà, il Rinaldi riproponeva in chiave moderna l'antitesi di Marziale fra "pagina lasciva" e "vita proba" rivendicando con ironia pungente e provocatoria l'inganno ipocrita della letteratura: "Pur inganna la lingua e l'occhio mente; / ché tra le nevi il cor, tra 'l gielo stassi, / e se tratta d'amore, amor non sente". ${ }^{8}$ A venire qui sovvertito è il patto autobiografico di stampo petrarchista: il Petrarca di "che quanto piace al mondo è breve sogno" ( $R V F \mathrm{I}, 14)$ diventa ora "ché quanto di lei parlo è un'ombra, un sogno"; e la poesia non parla più a "chi per prova intenda amore" ( $R V F \mathrm{I}, 7)$, ma dichiara polemicamente che "se tratta d'amore, amor non sente". Su tutt'altro tono sarà invece, dieci anni dopo, l'ammissione di colpa del Rinaldi pentito: "Quando favellai in versi, l'amore di bella donna mosse la lingua e 'l desiderio di vedere costei esaltata ingannò il giudicio, allettandolo a publicar le cose dette" (Rinaldi, 1617, p. 199). Proprio il mito di Barbara rappresenta una linea di frattura nell'esperienza poetica e umana del Rinaldi. Quel mito poetico si lega alle foreste selvagge, alle tempeste invernali, ai lampi che squarciano la notte e alle fiammeggianti aridità estive come figure della passione opposte all'Aprile platonico e petrarchista. Ha giustamente osservato Luisella Giachino:

Tornando al nome BARBARA, esso pare a me prima di tutto la spia di una cifra stilistica, della lussureggiante opulenza barbarica e anticlassicistica della stagione barocca, di un'abbagliante vivacità metaforica e cromatica, non priva di una segreta tendenza alla dissipazione e all'autocombustione. (Giachino, 2003, p. 8)

8. Rinaldi, 1603, p. 4 (IV, vv. 12-14). È una dichiarazione programmatica che capovolge la critica contro gli inganni delle prostitute su cui insisteva ampiamente la satira anonima: "Dunque credete voi che sian rubini / le labbia sue, non le vedendo smorte? / Opre son di cinabri e di verzini / che ingannano con l'arte e col lavoro / gl'inaveduti amanti" (Or si Rinaldi, che la Musa vostra, vv. 29-33). 
Barbara incarna l'emblema polemico di una poesia che canta la "vita dei sensi vitale", le "ombre" effimere del sensibile rischiarate dal "fuoco" arido dell'arguzia e dell'analogia. ${ }^{9}$ Viene così a delinearsi un percorso verso il basso e il confuso in cui il poeta, per trovare un frammento di luce, "s'accieca / nel fosco orror de la terrena ecclisse". ${ }^{10}$ Con le Rime nuove del 1603 il Rinaldi sperimenta un ritorno ardito e innovativo alla poetica giovanile dei Madrigali del 1588, ritrattata e ridefinita già nella Parte terza del 1590 sulla base di una "guerra" di stampo platonico tra "senso" e "ragione", forse anche per conformarsi alla linea culturale della neonata Accademia dei Gelati:

Duri carmi talvolta ed aspre note vergai cantando, e' $l$ piè girai sovente per calle umil, né mai s'alzò la mente, sì le strade al salir mi furo ignote. ${ }^{11}$

Ma facciamo ancora un passo indietro e ripercorriamo il "calle umil" del giovane Rinaldi a contatto con i grandi pittori bolognesi di fine Cinquecento.

\section{Nella "stanza" dei Carracci}

Fin da giovane il Rinaldi fu di casa nella "stanza" di Ludovico, Agostino e Annibale Carracci, frequentata non solo da "letterati", "scienziati" e "virtuosi d'ogni genere", ma anche da semplice "gente lieta e festosa", per cui, secondo Cesare Malvasia, "tante e tali erano le facezie, le novelle, le baie e le partite che, temperate da una continua allegria, le difficoltà dell'Arte o non si conoscevano o non si stimavano". ${ }^{12}$ Anche Giovanni Pietro Bellori ricorda che Annibale Carracci "fra gli studi delle arti più gravi mischiava le burle e le piacevolezze" ed "era destro nello spiegare arguzie e motti con parole". ${ }^{13}$ Era un esercizio di vivacità e di acutezza tutt'altro che estraneo all'inventiva di pittori ingegnosi e

9. A fare da sfondo al canzoniere per Barbara (Rime nuove I-LXIX) vi è sempre un risentito 'tu' polemico: "Luci vittrici e fulminanti stelle, / mirale tu, fa' tu del fallo emenda, / tu che chiami la donna un sesso imbelle!" (Rinaldi, 1603, p. 36).

10. Cfr. Rinaldi, 1590, p. 2. Ė una poetica dell'“informe" dichiarata già nel 1601: "Altro non è il mio stil ch'un mondo informe; / lasso, chi lo distingue? e chi scatena / da la notte e dal giel la fiamma e 'l giorno? / Mille d'alti desir superbe forme / erger vedriansi, e far lucida scena / d'onor lampadi mille ai poli intorno" (Rinaldi, 1601, p. 97). Non è improbabile che il Rinaldi avesse presente l'affresco raffigurante la Separazione della luce dalle tenebre dipinto dal giovane Guido Reni nel 1599.

11. Cfr. Rinaldi, 1590 , p. 105. Con la Parte terza del 1590 il Rinaldi inaugurava il canzoniere per Alba, ossia la nobildonna Pellegrina Capello Bentivoglio, e lo svolgeva poi con sempre meno convinzione nella Parte quarta del 1591, nella Parte quinta del 1594 e nella Parte sesta del 1598 in cui figurano ormai ben pochi richiami ad Alba e ai miti idillici del petrarchismo platonizzante. D'altro canto, se il sonetto assume un ruolo dominante di autoanalisi sentimentale dalla Parte terza fino alla Parte quinta, la Parte sesta risulta invece composta solo di madrigali dedicati a una pluralità di figure femminili.

12. Cfr. Malvasia, 1678, t. I, p. 471. Stando al Malvasia, il giovane Rinaldi fu "grande amico" di Agostino Carracci (t. I, p. 361) e il "diletto" di Ludovico Carracci (t. I, p. 481).

13. Cfr. Bellori, 1672, p. 75. 
avveduti come i Carracci, secondo cui l'eccellenza di un'immagine consiste nel raffigurare così vivacemente le contrastanti passioni di una scena al punto da suscitare la parola ammirata e partecipe di chi la guarda. ${ }^{14}$ Anzi, ha osservato Marc Fumaroli che, alle soglie dell'età barocca, negli studi dei pittori e nelle gallerie di quadri dei palazzi nobiliari l'arguzia delle immagini si riflette e si prolunga nella replica arguta ed epigrammatica del poeta che cerca nel dipinto un senso intimo e personale definendo così la propria singolarità di moderno:

Luogo destinato alla "civil conversazione", alla schermaglia arguta, una galleria principesca ornata di dipinti e statue si prestava a questi "scherzi" ingegnosi, e offriva loro i più fastosi pretesti. Se Fulvio Orsini, l'erudito al servizio della famiglia Farnese, apprezzava, come sappiamo, l'arte dell'epigramma, Annibale Carracci, che Bellori descrive come innamorato di senechiana brevitas, e quindi delle "punte" di Marziale, non poteva che aderire a quella tendenza di sapore moderno e mondano. (Fumaroli, 1995, p. 79)

Con la crisi dell'enciclopedismo rinascimentale e la crescente specializzazione dei saperi, a detta del Fumaroli emerge un nuovo rapporto di convergenza e continuità tra immagini, suoni e parole entro spazi semiprivati di libera sperimentazione artistica e culturale ai margini dell'ufficialità. Negli ambiti più diversi s'impone man mano la ricerca di un punto di fuga al di là delle singole arti e delle forme sensibili tale da far desiderare all'anima la nascosta armonia spirituale che è comune alla pittura, alla musica e alla poesia. ${ }^{15}$ Non può certo essere solo un caso che i madrigali giovanili del Rinaldi prendano forma a contatto con le arguzie pittoriche dei Carracci. ${ }^{16}$ Nella misura breve

14. Celebre è l'aneddoto tramandato da Giovanni Battista Agucchi: "Un letterato de' primi di quel tempo, domandò ad Annibale, chi si fosse portato meglio di due Pittori della sua Scuola in un lavoro, che insieme fecero per un Cardinale, cioè un'Historia grande per ciascuno della vita di un medesimo Santo, dipinte a fresco in Roma dentro una Chiesa nelle due lati, l'un incontro all'altro. Al quale quesito Annibale rispose, che quelle due Historie erano state cagione, che egli si era conosciuto se stesso per un grandissimo balordo: perché non haveva mai saputo comprendere, quale di esse meritasse d'esser più lodata; sintanto che egli non imparò a conoscerlo da una Vecchierella; la quale havendo per mano una Fanciulla, si fermò un giorno a guardare l'una e l'altra di quelle Historie, \& egli l'osservò; che mentre ella ad una fissò lo sguardo, andò voltando l'occhio da ogni parte per mirarla tutta, ma non disse mai una parola, né diede altro segno d'alcun affetto, che in lei havesse cagionato il guardar quella Pittura; ma poi all'altra Historia voltatasi, cominciò a dire alla Fanciulla: Vedi vedi figlia quell'huomo, che fa la tal cosa; e col dito gli accennava la Figura, che quell'attione, ch'ella diceva, rappresentava: e così di mano in mano mirando l'altre Figure, le additava, e ne dichiarava con gusto le attioni alla Fanciulla, la quale ancora pareva che se ne prendesse diletto. Hor vedete (disse Annibale al Letterato) com'io ho imparato a conoscere, quale delli nostri due Dipintori habbia più vivamente espressi gli affetti, e più chiaramente la sua Historia dichiarata" (Guido Reni 1575-1642, 1988, p. 211).

15. Cfr. Fumaroli, 1995, pp. 13-28, 61-80 e 259-458.

16. Fra l'altro Agostino Carracci faceva professione di erudito e letterato "componendo sonetti, madrigali e sestine in modo che il Rinaldi, suo grande amico, avesse più volte a dire comporre egli meglio di lui" (Malvasia, 1678, t. I, p. 361). Ad Agostino il Rinaldi dedica un dittico di sonetti nella Parte quarta delle Rime, Mentre involi a Natura e doni ad Arte e Carraccio in nobil carro accesa mente (Rinaldi, 1591, pp. 76-77). Sul concetto di "scherzo" 
e ingegnosa del madrigale, la poesia si riappropria della musica, del disegno, del colore, del teatro, del mito, gareggiando argutamente con i materiali più diversi in una sorta di estemporanea polifonia delle arti. Eppure i Madrigali del Rinaldi sono tutt'altra cosa dalla Galeria che Giovan Battista Marino pubblicherà trent'anni dopo. Proviamo allora a considerare più in generale l'incontro tra cultura figurativa, tradizione ecfrastica e poesia lirica chiedendoci che cosa lo abbia reso possibile nel quadro del mutamento di paradigmi espressivi tra Rinascimento e Barocco.

Per il classicismo rinascimentale la perfezione di un dipinto non consisteva solo nel riprodurre le armoniose forme esterne di un evento o di un volto, ma anche nel rendere evidente la sua dimensione ideale e interiore. Affermare con Plutarco che la poesia è una pittura che parla e la pittura una poesia che tace, voleva dire prescrivere alla pittura l'arduo compito di esprimere i valori dell'animo e i moti segreti dell'emozione con le sole risorse del disegno e del colore, educando così l'occhio dell'osservatore ad andare al di là delle apparenze sensibili. Vi era in ciò il proposito di gareggiare con l'arte degli antichi riguardo a cui Marziale aveva appunto esclamato: "Ars utinam mores animumque effingere posset! / Pulchrior in terris nulla tabella foret". ${ }^{17}$ In un celebre dittico di sonetti, Petrarca aveva elogiato Simone Martini per aver ritratto non solo le "membra" e la "figura" di Laura, ma la sua "beltà" interna contemplata forse "in paradiso", con occhi del tutto sgombri dal "velo" della materia, tanto da giungere a raffigurarla compiutamente in un" "opra gentile", "di quelle che nel cielo / si ponno imaginar, non qui tra noi" (RVF LXXVII-LXXVIII). È una fiducia nella capacità della parola poetica di esprimere e padroneggiare le mute profondità interiori dell'immagine che tende a farsi convenzionale e problematica nel petrarchismo di pieno e tardo Cinquecento. Non è certo per una semplice variazione del modello che Giovanni Della Casa riscrive a rovescio quella coppia di sonetti petrarcheschi dichiarandosi incapace di assolvere il proprio compito di poeta dinanzi al perfetto illusionismo del ritratto di Elisabetta Quirini dipinto da Tiziano: "Ma io come potrò l'interna parte / formar giamai di questa altera imago, / oscuro fabro a sì chiara opra eletto?" (Rime XXXIII-XXXIV). Per il Casa, infatti, non è più possibile "trovar" ed esprimere il "vero" intimo dell'immagine nella sua eletta unicità spirituale, ma il poeta non può fare altro che trascrivere i molteplici effetti emotivi che essa produce nel suo animo turbato e partecipe di spettatore (Rime XXXIV). Non che la prosa ecfrastica rinascimentale da Pietro Aretino a Giorgio Vasari non avesse prestato attenzione anche agli effetti che l'opera d'arte intende suscitare, ma li considerava appunto come riflesso consapevole della genialità dell'artista e non come reazione affettiva o intellettuale del soggetto che guarda e che,

pittorico si veda anche Pepper, 1983, pp. 72-74

17. Marziale, 2007, p. 568 (X, 32). Un senso di emulazione trionfante traspare ad esempio dai sonetti di Pietro Bembo sul ritratto di Maria Savorgnan realizzato da Giovanni Bellini con tale abilità da "mores animumque effingere" (Rime XIX-XX): "O immagine mia celeste e pura, / [...] / credo che 'l mio Bellin con la figura / t'abbia dato il costume anco di lei" (Bembo, 1997, pp. 521-523). 
leggendo man mano l'immagine, interroga e definisce il proprio particolare orizzonte di senso. ${ }^{18}$ Invece, allorché il giovane Torquato Tasso riprende e capovolge il Casa risentito e afflitto di Son queste, Amor, le vaghe trecce bionde (Rime XXXIV) nell'estasi felice del sonetto Son queste, Amor, le vaghe chiome d'oro (Rime CCXVII), come se uno stesso dipinto potesse produrre in persone diverse emozioni anche opposte, è evidente che la prospettiva petrarchesca risulta ormai completamente rovesciata e il punto di fuga dell'opera d'arte non consiste più tanto nei valori spirituali che traspaiono tacitamente dall'interno delle sue figure, ma risiede piuttosto nell'occhio esterno che la guarda e la interpreta a partire dalla propria singolarità individuale. Ciò che conta non è solo il significato che si esprime nell'immagine, ma l'effetto che si imprime nell'animo dello spettatore, secondo angolature sempre particolari per cui anche ciò che è finto e illusorio può ispirare paradossalmente sentimenti veri e autentici. ${ }^{19} \mathrm{E}$ una concezione nuova che non solo rende pienamente possibile l'ipotesi di un'ecfrasi su base lirica, ma che ha la forza espansiva di una scoperta fondamentale al punto che il Tasso la riprende e la sviluppa al centro del giardino incantato di Armida quando Rinaldo teorizza argutamente la soggettività del bello affermando che solo il suo sguardo acceso di passione, e non l'immagine riflessa nello specchio, può essere il "ritratto vero" delle bellezze della maga:

- Volgi, - dicea - deh volgi - il cavaliero

- a me quegli occhi onde beata bèi,

ché son, se tu no 'l sai, ritratto vero

de le bellezze tue gli incendi miei;

la forma lor, la meraviglia a pieno

più che il cristallo tuo mostra il mio seno. (Ger. lib. XVI, 21)

Quello prefigurato ora dal Tasso è in fondo il crinale della modernità e non sorprende che la scuola dei Carracci abbia sentito l'esigenza di misurarsi con quella scena ingegnosa e decisiva della Gerusalemme liberata. ${ }^{20}$

Ogni novità ha però bisogno di un tempo d'assestamento. Non v'è dubbio che la prima esperienza lirica del Rinaldi si muova fra il Tasso e il Casa, ed è già indicativo il fatto che nei Madrigali abbia ampio sviluppo il gioco arguto fra "vero" e "falso", fra "vera beltà" e "finta imago", pur nel segno di un compromesso con l'eredità petrarchista:

Ch'incenda un'alma e strugga

vera beltà d'angelica figura,

18. Sul nuovo senso barocco dell'individualità si veda Forni, 2018, pp. 9-32.

19. Per la rilevanza dell'illusione soggettiva nella poesia tassiana si rimanda a Forni, 2019, pp. 78-86. Sul dittico del Casa e il sonetto del Tasso giova fare riferimento a Di Benedetto, 1996, pp. 143-153, e Pich, 2007.

20. Basti qui il rinvio al Rinaldo e Armida di Lodovico Carracci (1593) e all'analoga tela di Annibale Carracci (1601) oggi al Museo di Capodimonte a Napoli. Sull'importanza del Tasso nella cultura figurativa bolognese tra Cinque e Seicento si veda almeno Raimondi, 1995, pp. 21-53. 
tutt'opra è di Natura;

ma ch'una finta imago

arda con mille e mille fiamme un core,

quest'è tua forza Amore.

Fiero e potente Mago,

se vuoi ch'ascoso porti entro al mio seno

di non viva bellezza un vivo ardore,

deh mi concedi almeno

che nel bel simulacro appaghi anch'io,

novo Pigmalione, il desir mio. (Rinaldi, 1588, p. 74) (1 $^{21}$

Qui è Amore, cioè la magia della passione e della poesia, che dà vita all'immagine. Di per sé la perfetta resa naturalistica di un dipinto non genera appagamento ed è solo la dimensione affettiva di chi guarda che può dare valore all'arte:

Al natural pingesti,

dotto pittor, l'imago,

per far del dolce obietto il mio cor pago;

ma incauto non scorgesti

ch'in vece di gradire,

sortì contrario effetto al tuo desire.

O mirabil successo!

tornò crudel quell'atto

che per pietà fu fatto. (Rinaldi, 1588, p. 124)

Pur riprendendone l'accorato patetismo, vero è che i Madrigali del Rinaldi restano al di qua del confine prospettato dal Tasso e tendono ancora a ribadire il primato della poesia sulla pittura senza mai cimentarsi in un'effettiva ecfrasi lirica. Negli anni in cui i pittori bolognesi gareggiavano nel dare all'incarnato una piena illusione di vita, il Rinaldi non trova di meglio che esprimere i valori dell'animo con un'arguta spiritualizzazione emblematica dei colori: il bianco per la Fede, il rosso fiammeggiante per l'Amore... ${ }^{22}$ Tutt'al più i madrigali giovanili piegano all'autonomia del linguaggio lirico la tradizione dell'emblema ${ }^{23}$ e dell'epigramma ecfrastico:

Pittor, tu che mi mostri del Sol l'imagin finta, non sai ch'altri nel cor me l'ha dipinta?

L'oro, le perle e gli ostri

m’impresse Amor nel petto;

quel che dipingi tu, d'Arte è pittura, quel ch'io porto nel seno, Amor figura:

scopri l'amato obbietto,

21. Cfr. la chiusa di RVF LXXVIII, 12-14: "Pigmalion, quanto lodar ti dêi / de l'imagine tua, se mille volte / n'avesti quel ch'i' sol una vorrei".

22. Cfr. Perini, 1995, pp. 125-126. È un simbolismo dei colori che si rifa all'emblema 118 di Andrea Alciato, In colores.

23. Cfr. Ritrovato, 2005, pp. 46-55. 
scoprilo a me pittor, e si consumi

per Arte e per Amor il cor e i lumi. (Rinaldi, 1590, p. 114)

Per intendere appieno la sfida scherzosa del madrigale, occorre dire che "del Sol l'imagin finta" allude sì a un ipotetico ritratto dell'amata, ma anche al motivo dell'impossibilità di raffigurare appieno il fulgore del sole quale si legge ad esempio in uno degli epigrammi di Celio Calcagnini sul ritratto di Ercole I d'Este dipinto da Dosso Dossi:

ICONICA HER.[CULIS] D.[UCIS] IMAGO

Exprimit in tabula Phaëthontem Cous Apelles,

Sed lucem et radios non potis exprimere.

Sic tua, mi princeps, Dossus forte exprimit ora,

Virtutem et mores non potis exprimere. ${ }^{24}$

Quella del pittore resta una "imagin finta" se il poeta non vi aggiunge la parola, la luce dell'interiorità, e la conclusione pungente del madrigale sfida appunto il pittore a svelare l'oggetto amato nella sua interezza di corpo e anima, in modo che nel medesimo tempo gli occhi siano consumati dalla maestria dell'Arte e il cuore da Amore. Al pittore non si chiede tanto di "esprimere" le virtù interiori attraverso l'immagine, ma piuttosto di mostrare ciò che "impresse Amor nel petto". Va da sé che, a tali condizioni, l'ecfrasi lirica non può andare oltre l'evocazione di un'immagine mentale.

Proprio il primato della poesia circoscrive il campo autoreferenziale del discorso lirico. In apertura della seconda parte dei Madrigali il poeta si presenta come una sorta di statua immaginaria:

$\mathrm{O}$ tu, che miri? Uom fui;

or, colpa di colei che mi trasforma,

pietra son di materia e non di forma;

e se forse al sembiante

sembro quel ch'ero innante,

fisa gli occhi e vedrai

- se ben vi miri, ahi lasso -

ch'ove già fui di carne, or son di sasso.

Pur l'amo ancor; s'altri tel chiede mai,

tu digli, e dirai vero,

che mutai spoglia e non mutai pensiero. (Rinaldi, 1588, p. 127)

Nell'invito a osservare bene e nella prosopopea dialogica è evidente il richiamo all'epigramma ecfrastico tardoantico e in particolare agli epigrammi sulla statua di Niobe scolpita da Prassitele, ${ }^{25}$ ma quei riecheggiamenti rendono ancora più significativo il fatto che l'opera d'arte descritta sia ora la figura stessa del poeta,

24. Pigna, Calcagnini, \& Ariosto, 1553, p. 208. Calcagnini attualizza un motivo dell'epigramma ecfrastico tardoantico: si veda ad esempio Antologia greca, XVI, 32 e 77.

25. Cfr. Antologia greca, XVI, 129-134; Ausonio, Epigrammaton liber, 51; Pigna, Calcagnini, \& Ariosto, 1553, pp. 208 (De Niobe), 210 (De lapide sipyleio) e 215 (Niobe). Per la fortuna rinascimentale degli epigrammi antichi sulla Niobe di Prassitele si veda Ruiz Sánchez, 1998. 
solo simbolicamente trasformata in sasso e costante nell'amore come Niobe nel pianto. L'io moderno resta il punto focale dell'arguzia lirica. ${ }^{26}$

Man mano però che l'eredità petrarchista si esaurisce attraverso lo sviluppo del canzoniere per Alba, il Rinaldi sperimenta nuove possibilità tanto che, dieci anni dopo i Madrigali, la Parte sesta delle Rime mostra un ritorno d'interesse per il madrigale iconico e descrittivo. Non è un caso che, al posto della sfida a un generico pittore, compaia qui l'elogio senza riserve di due pittrici moderne ingegnosamente rappresentate all'opera dinanzi al loro cavalletto:

\section{Lavinia Fontana Pittrice}

Chi mai non vide Amore

e'l desia pur veder che parli e spiri,

al tuo pennello il chieggia, al tuo colore;

tosto fia ch'egli il miri,

né già di senso privo,

ché se tu pingi Amore, Amor è vivo.

\section{Fede Gallizia Pittrice}

Vaga fedel pittrice,

se talor folle e vano

amator pingi e miri,

han diverso pensier gli occhi e la mano;

questa col dotto stil fa ch'egli spiri;

quei co' bei raggi accensi

gli furan l'alma e i sensi;

tal che 'n un punto (ahi lasso)

tu 'l formi in tela e lo trasformi in sasso. (Rinaldi, 1598, pp. 98-99)

Non vi sono più colori astratti e simbolici, ma il "tuo colore", il "tuo pennello", la "mano" concreta dell'artista. Il poeta non è ancora lo spettatore diretto di quadri reali, ma essi entrano già di sbieco nel gioco dell'invenzione poetica se si coglie il riferimento alle vivaci mitologie erotiche di Lavinia Fontana o alla fama di Fede Galizia come prodigiosa ritrattista. Nel contatto vivo con i pittori del suo tempo, il Rinaldi giunge ad attraversare la soglia tra l'affinamento espressivo di un consolidato repertorio sentimentale e un'arte impressiva dell'immagine estrosa e icastica, diventando così uno dei primi poeti a oltrepassare il confine tra l'autunno manierista del petrarchismo rinascimentale e la nuova epoca barocca. ${ }^{27}$

26. Non è improbabile che con quel madrigale il Rinaldi intendesse dare una risposta ai rifacimenti antiquari di Luigi Groto apparsi l'anno prima e fra i quali figuravano anche una Scoltura di Medusa e una Niobbe: cfr. Groto, 1587, pp. 138 e 147 (Fui Niobbe, indi in sasso mi cangiai).

27. Non si tratta però di leggere quell'attraversamento soltanto nei termini involutivi di una perdita di levità e di scioltezza come propone, in una sintesi pur lucida e perspicace, Ritrovato, 2005, pp. 86-87: "Da una poesia d'ingegno, più leggera nei temi e nella forma, rubricata sotto l'insegna della lirica d'amore [...] a una poesia mossa essenzialmente a stupire il lettore con dispiegamento di artifici retorici in cui prevale l'ardita fattura delle immagini; 


\section{3. "Pittura e Poesia suore e compagne"}

Torniamo ora ad aprire le Rime nuove del 1603 per andare oltre la sezione dedicata a Barbara e considerare la seconda parte encomiastica e funebre del volume in cui trovano posto alcune fondamentali dichiarazioni di poetica e soprattutto una serie di componimenti ecfrastici su opere d'arte realmente esistite. Non si tratta certo di un esito ovvio o spontaneo se si considera che per quasi vent'anni il Rinaldi aveva frequentato gli studi dei pittori e le collezioni d'arte dei palazzi aristocratici senza mai porsi davanti a un dipinto in veste di poeta. Per arrivarci era necessario mettere da parte il primato della poesia come autonoma espressione di valori spirituali e concepire le diverse arti sul medesimo piano in quanto "suore e compagne". Solo così la parola lirica può interrogare l'effetto e il significato di un dipinto...

Nel 1602 il cardinale Bonifazio Bevilacqua Aldobrandini aveva acquistato dalla famiglia Campeggi la splendida Villa del Tuscolano nei pressi di Bologna e fra i quadri che ornavano la galleria vi era una piccola Pietà di Dosso Dossi celebrata dal Rinaldi in un sonetto al nuovo proprietario:

Ad una Pietà del medesimo, Opera del Dossi.

Bella PIETÀ, cui sì penosa i' miro, qual dotto stil, qual saggia man ti finse?

E chi d'aspre ritorte, ohimè, t'avvinse,

bella Pietà? tua doglia è mio sospiro.

Christo nel duol, ne l'arte il Dossi ammiro,

che 'l nostro error nel tuo livor dipinse;

Pietà figlia d'Amore, Amor ti spinse

al penar, la tua pena è tuo desiro.

Cortesia di Signor non mi ti neghi,

cara imago vital, lucida vita,

su l'altar del mio cor t'offro i miei preghi.

Bella PIETÀ, che m’apri aurea salita, quel nodo, che te cinge, anco me leghi;

specchio a gli occhi, al cor cibo, a l'alma aita. (Rinaldi, 1603, p. 78)

Ora, essendo il tema del Cristo morto assai raro nell'opera del Dossi, non è difficile identificare il dipinto a cui il sonetto fa riferimento. Nella Fototeca della Fondazione Federico Zeri di Bologna si conserva infatti la riproduzione a colori di una Pietà del Dossi, oggi d'ignota ubicazione, che risulta del tutto coerente con le indicazioni poetiche del Rinaldi e anzi le illumina di un senso più esatto e più ingegnoso. ${ }^{28}$ Osserviamo dunque il quadro a paragone del sonetto. Fra le penombre della notte incipiente spicca in primo piano il busto

in altre parole, da una poesia d'occasione che mira a una raffinata stilizzazione della forma entro una limitata ma vivace gamma di temi [...] a una poesia di gareggiamento che mira invece alla rivelazione fragorosa del tema, alla sua fastosa celebrazione metaforica: questo è, in sintesi, il percorso poetico di Cesare Rinaldi”.

28. Bologna, Fondazione Federico Zeri, Fototeca, Fondo Zeri, collocazione PI_0422/3/2, disponibile su http://catalogo.fondazionezeri.unibo.it/scheda/fotografia/96567/. 
del Cristo morto avvolto in un drappo purpureo e sorretto dalla Vergine in modo che i due volti di tre quarti appaiono alla stessa altezza inclinati l'uno verso l'altro e, in basso, anche la mano destra e forata del figlio ricade in posizione perfettamente speculare rispetto a quella della madre che ne sta coprendo le nudità con un lembo della stoffa. Proprio l'accentuata simmetria della composizione rende più evidenti le differenze tra le due figure: la fronte di Cristo è macchiata di sangue per la corona di spine (le "aspre ritorte" del v. 3), quella della Vergine è invece coperta da una cuffia candida in segno di purezza; gli occhi di Cristo sono chiusi nell'abbandono della morte, quelli della Vergine lo guardano arrossati e lucidi dal pianto; ma ciò che ha più efficacia è il contrasto di tonalità fra il colore livido e freddo del corpo di Cristo (il "livor" del v. 6) e la rosea vitalità del volto e della mano della Vergine che cinge e sostiene Cristo fra le sue braccia (il "nodo" del v. 13). Ora, se si commisura il dipinto alla sua trasposizione poetica, appare subito evidente che il sonetto non si limita a descrivere l'immagine, ma piuttosto la interroga in cerca di un senso personale. Al centro del discorso lirico vi sono le reazioni interiori del poeta in presenza del quadro, mentre lo osserva ammirato, tant'è vero che egli può tenere conto insieme del soggetto (la "bella Pietà") e dei dettagli concreti della sua esecuzione (la "saggia man"). Ecco allora che il dipinto diventa esperienza intima di verità, punto d'origine della parola poetica ("tua doglia è mio sospiro"), al punto che persino una specifica scelta di colore può essere assunta soggettivamente come simbolo ingegnoso dell'intera storia umana ("ne l'arte il Dossi ammiro, / che 'l nostro error nel tuo livor dipinse"). Non vi è più una "finta imago" rispetto ai valori superiori della poesia, ma una "imago vital" e anzi una "lucida vita" da svolgere al proprio interno ("su l'altar del mio cor") con l'aspirazione devota ad essere legato dal medesimo "nodo" che cinge Cristo e che è al contempo quello delle "aspre ritorte" della Passione e dell'abbraccio pietoso della Vergine. Né parrà forse una sottigliezza l'ipotesi che proprio l'orlo dorato del drappo con cui la madre sta avvolgendo il corpo del figlio possa avere suggerito l'idea dell' "aurea salita" al di sopra del dolore e della morte. Nell'ecfrasi emotiva e arguta del Rinaldi vi è un'interiorizzazione lirica dell'immagine per cui ogni dettaglio del quadro può divenire "specchio a gli occhi, al cor cibo, a l'alma aita". Quel che più conta nell'opera d'arte è ciò che essa, per così dire, imprime nel petto.

Ma non solo. Nelle Rime nuove figura anche un sonetto sopra un dipinto del giovane Guido Reni, un Noli me tangere oggi disperso e realizzato per il Rinaldi stesso, forse poco prima che il Reni partisse per Roma nel 1601 o al suo ritorno nel 1603. Ha certo ragione Salvatore Ritrovato quando osserva che il Rinaldi "spinge il lettore a guardare oltre la figura" della Maddalena per cogliere il "dilemma angoscioso del suo sentimento nascosto" e la "silenziosa intensità di un amore impossibile" (Ritrovato, 2005, p. 35). Né certo il cantore di Barbara poteva rimanere estraneo all'accorato sentimento d'amore della "santa peccatrice". ${ }^{29} \mathrm{Ma}$ quel che desta ancora più interesse è che il sonetto

29. Cfr. al riguardo Piantoni, 2013. 
non descrive tanto il quadro, quanto l'atto di dipingerlo, l'ispirazione creativa del pittore che dialoga con la figura a cui dà forma. Ascoltiamo dunque il testo:

NOLI ME TANGERE, Opera di Guido Reni.

Nel peccar fiamma, e nel pentirsi foco

fu Madalena; al vero amante intesa

Guido la pinge, e pallida et accesa

co' sospir dàlle un suon tremante e fioco.

Bella di Christo amica, un cor è poco

a sì alto dolor; ma che ti pesa?

che 'l tuo amator ti spregi? ov'è l'offesa,

ivi è lo scampo, in amor l'ira è gioco.

Mentr'ei da sé t'arretra, a sé ti chiama,

ch'un foco è del tuo incendio; o pittor saggio,

dille che tanto ei vuol, quanto ella brama.

Pur faccia umida nube onta al bel raggio,

e per piegar chi le si piega et ama,

sia lo squarciato crin del cor messaggio. (Rinaldi, 1603, p. 95) ${ }^{30}$

Non solo Guido dipinge la Maddalena scossa e turbata, ma con i suoi "sospir" di devozione quasi le dà voce, le presta una parola ancora incerta e indistinta, "un suon tremante e fioco". Entra allora in gioco il poeta per interrogare l'immagine della donna e intendere il contrasto di sentimenti che anima la sua fisionomia agitata e afflitta. Qui davvero il poeta fraternizza con il pittore nel definire il senso particolare della scena e nel definirsi attraverso di essa. Vi è una sorta di dialogicità interiorizzata, quasi da esercizio spirituale se non fosse che l'approfondimento riflessivo delle domande e delle risposte non conduce al trionfo del trascendente, ma piuttosto all'affermazione piena, totalizzante del sentimento. Va da sé, osserva il poeta, che un singolo amore umano non possa bastare all'amore universale di Cristo testimoniato con la Passione ("un cor è poco / a sì alto dolor"), ma per quale ragione allora la Maddalena appare così affranta e avvilita? forse si sente respinta? Non dovrebbe perché "offesa" e "scampo", "ira" e "gioco", "peccar" e "pentirsi" fanno parte dello stesso "foco" d'amore. Più che le cadenze mondane di una "schermaglia d'amore", ${ }^{31}$ bisogna riconoscere qui un fondamentale assunto teologico dell'Epistola ai Romani: "ov'è l'offesa, / ivi è lo scampo" ricalca infatti "Ubi autem abundavit delic-

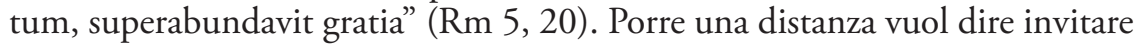
ad attraversarla ("Mentr'ei da sé t’arretra, a sé ti chiama") in quanto Cristo è un fuoco d'amore non diverso da quello che arde la Maddalena ("ch'un foco

30. Non è improbabile che il Rinaldi avesse manifestato un interesse o una predilezione per tale tema visto che, parecchi anni dopo, il Reni fece dono al poeta di un altro dipinto di analogo soggetto: "la Maddalena fatta al suo dilecto Rinaldi, per dargli a divedere quanto avvantaggiato ei si fosse da que' primi tempi, ne quali avevagli colorito il Noli me tangere, celebrato nelle sue rime stampate, in quel sonetto: Nel peccar fiamma, e nel pentirsi foco, etc." (Malvasia, 1678, t. II, p. 42).

31. Piantoni, 2013, p. 39: "la coppia Cristo/Maddalena sembra darsi a una tipica schermaglia d'amore". 
è del tuo incendio"). Così il poeta suggerisce infine al pittore che cosa dire alla donna per consolarla: lei e Cristo vogliono la stessa cosa, condividono lo stesso incendio d'amore ("o pittor saggio, / dille che tanto ei vuol, quanto ella brama"). Ma proprio per questo il sentimento umano, le lacrime, i gesti di dolore della Maddalena mantengono una piena validità come "messaggio" del cuore, in grado di rendere evidenti le verità profonde, invisibili dell'amore. E davvero qui pittura e poesia instaurano una nuova familiarità, un confronto alla pari e quasi un'intesa fra arti sorelle: "Pittura e Poesia suore e compagne, / che quei ch'è gran pittore, è gran poeta [...]". ${ }^{32}$ Quel confine è ormai varcato.

Qualcosa di analogo potrebbe dirsi anche per i tre sonetti e i dieci madrigali delle Rime nuove dedicati a un dipinto di Pietro Faccini, dapprima allievo e poi orgoglioso rivale dei Carracci. ${ }^{33}$ Purtroppo non disponiamo più del quadro né sono rimaste altre testimonianze di esso al di fuori dei componimenti ecfrastici del Rinaldi. Era un'opera probabilmente di piccole dimensioni che raffigurava Amore legato a un mirto e Venere che lo batte con una sferza fatta di rose. Ha certo ragione Giovanna Perini quando ipotizza che quel quadretto mitologico potesse essere una "risposta" del Faccini a una celebre stampa di Agostino Carracci tratta dalla serie delle Lascivie, il Satiro che batte una ninfa (Perini, 1995, p. 124); tuttavia, nel rifarsi alla tradizione del 'Cupido punito' da Ausonio e Petrarca fino al Rinascimento, il Faccini doveva avere in mente anche un'altra incisione del "libretto di scherzi" delle Lascivie: la tavola Venere punisce l'Amore profano, in cui Cupido bendato viene tenuto fermo da un fanciullo mentre Venere lo percuote con un fascetto di verghe, in modo tale però che il volto dell'Amore profano risulta paradossalmente rivolto verso il cielo e quello di Venere, coperto d'ombra, è chino verso terra. ${ }^{34}$ Come nel Noli me tangere del Reni, anche qui due differenti figure dell'Amore appaiono divise da una distanza o una disimmetria complessa e sfuggente, tanto che l'osservatore può stabilire fra di esse una precisa scala di valori solo a partire dalla propria sensibilità, dall'acutezza delle domande che rivolge all'immagine e quindi in base alla propria variabile esperienza del mondo. Ed è allora significativo che fin dal primo sonetto sopra l'ingegnosa ed elusiva scenetta mitologica del Faccini, il Rinaldi si ponga di fianco al pittore e in dialogo con il dipinto nel suo farsi:

Al MED.[ESIMO] SOPRA UNA PITTURA

FACINI, egli è tua gloria il tuo pennello,

32. Rinaldi, 1603 , p. 105 . Ma si veda anche la tarda lettera al Reni del 23 febbraio 1619: "Io non trovo cosa più maravigliosa della Pittura; ella è un'Arte che tutte l'arti in sé comprende; un'Arte che tanto può, quanto vuole; io non dico ch'ella abbia forze uguali alla Natura, ché la Natura crea e la Pittura imita le cose create; con tutto ciò i Pittori eccellenti sono così rari imitatori che l'imitazione (se credi agli occhi) è creazione" (Rinaldi, 1620, pp. 141-142).

33. Cfr. Rinaldi, 1603, pp. 107-114.

34. Cfr. De Grazia, 1984, p. 172, e Malvasia, 1678, t. I, p. 97: "Un'altra [Venere nuda] che, fatto prendere a cavallo ad un puttino Amore bendato et alato, con verghe alla mano lo flagella, piangendo un altro a cavallo del turcasso, in bel paese". 
e la tua gloria è cote al mio diletto; pingi sferzato Amor, pingilo stretto a un mirto e Citerea vibri il flagello. Sia di rose la sferza; il crudo, il fello oda rimproverarsi il suo difetto; seco è pietà l'impoverir d'affetto, non ti lusinghi il volto umido e bello. Sento già le percosse, odo le strida. Ahi, cattivello Amor, di che ti lagni? Ogni commesso error la pena sfida. Ma mentre piagne Amor, tu seco piagni, Facin? non lo slegar, ch'ei non t'ancida; ché, se perdi pietà, luce guadagni. (Rinaldi, 1603, p. 107)

Ora, senza esaminare nel dettaglio la corona di sonetti e madrigali sul dipinto, basti dire che l'intento del Rinaldi è proprio quello di moltiplicare i punti di vista, le arguzie interrogative, i piani di lettura, i significati possibili della scena in bilico tra il serio e il faceto, tra la punizione morale dell'errore e una sorta di recita attraente e ironica di Venere che si diverte a trarre in inganno chi guarda, quasi a ribadire che "in amor l'ira è gioco":

Pingi sferzato Amore,

FACIN? di rose ha Citerea la sferza?

Ah, che poco pesanti i colpi affretta;

scherzo, ma non vendetta;

sì, ch'ella noi deride, e seco scherza,

ché, mentre irata sferza

con flagello di fior membra di latte,

le infiora, e non le batte. (Rinaldi, 1603, p. 108) ${ }^{35}$

Ed è un gioco libero e aperto del poeta con l'immagine che diventa ancora più evidente se si tiene conto di una delle probabili premesse iconografiche del quadro: l'incisione Amore sorpreso nei Campi Elisi dagli amanti di Giulio Bonasone, in cui sono le anime degli amanti a raccogliere mazzi di rose e fiori per percuotere Amore "scherzando", mentre Venere scende adirata dal cielo per punirlo in modo tutt'altro che giocoso. È ciò che dichiara anche l'epigramma inciso su una roccia in primo piano:

Volò ne' Campi Elisi un giorno Amore dove l'anime vivon degli amanti, le quai volendo vendicar l'ardore che dentro a' corpi avean patito inanti, corser piacevolmente con furore e lo legar co' suoi legami, e quanti sdegni, martiri e pianti avean sofferto gli ricordaro, e gli renderno il merto. E di rose e di fior mazi facendo

35. Sul motivo dell'ira e del riso rasserenante giova tenere conto anche del mito poetico di Barbara: "Barbara rise: - A che tant'ire, o sciocchi?" (Rinaldi, 1603, p. 30). 
lo battevan scherzando a gara insieme.

Scese Vener dal ciel, d'amore ardendo per Marte, e lo battea con forze estreme.

Sciolsero poi quell'anima ridendo et ei, che maggior mal futuro teme, spiegò l'ali, e fuggìssi, e tornò poi in questo mondo a trionfar di noi. ${ }^{36}$

Nella stampa del Bonasone lo "scherzo" delle anime defunte e la "vendetta" di Venere innamorata sono due azioni distinte e l'iscrizione provvede a precisarlo per aiutare l'osservatore a percorrere il disegno e decifrarne il significato. Invece, nel quadro del Faccini non solo è Venere a recare in mano il "flagello di fior", ma il senso della scena rimane indeterminato e ambiguo né vi è alcuna didascalia a chiarirlo. Mentre l'incisione del Bonasone presenta ancora lo schema dell'emblema rinascimentale per l'esatto rispecchiamento di epigramma e immagine, la sfasatura tra il dipinto del Faccini e l'ecfrasi lirica del Rinaldi introduce invece una voluta di possibilità in cui ciò che conta è l'effetto dell'opera su chi la osserva. Se, a detta del Fumaroli, l'arte sacra fra Cinque e Seicento prende a "giocare con le apparenze per rendere visibile la trascendenza" e tale ricerca dell' effetto spirituale consiste nell'additare un "punto di fuga" esterno al quadro che i pittori "fanno indovinare e desiderare all'occhio interiore senza poterlo mai mostrare" (Fumaroli, 1995, pp. 19, 24 e passim), vero è d'altro canto che l'arte di soggetto storico o mitologico mira a suscitare una reazione emotiva o intellettuale che ha come punto di fuga l'io di chi guarda il dipinto e vi cerca un significato prospettico, spiritoso, che definisca la singolarità individuale dello spettatore in forma di interrogazione di sé e di acutezza ingegnosa. Un sonetto, allora, può diventare davvero l'equivalente critico e soggettivo di un quadro.

Ma varcare il confine della modernità comporta altresì un controeffetto sullo statuto stesso della poesia. Non per nulla Benedetto Croce ha potuto considerare il Rinaldi come il primo e il più acceso "tra gli arditi futuristi del tempo" (Croce, 1928, p. 62). Nella sua ricerca impressiva e pungente dell'effetto, la parola poetica riconosce ora la propria affinità con le arti sorelle e si fa "dispensiera di lampi al cieco mondo" e "musico pennel che parla e pinge". ${ }^{37}$ Nella cultura postrinascimentale educata al linguaggio dei simboli, il quadro assomiglia sempre più a un emblema incompleto e aperto che indica lo spazio di un significato che è al di là dell'immagine e tocca all'osservatore intuire e rivivere. Analogamente, nelle Rime nuove del Rinaldi il sonetto diventa una composizione di immagini emotive e visionarie che hanno il loro punto di fuga nell'animo di chi le contempla. Era in fondo questo il segreto del "barbaro nome" che deve fare "corona a lo stil": l'impasto di diversi registri lessicali, lo

36. Cfr. Gabinetto dei disegni e delle stampe della Pinacoteca nazionale di Bologna, 1975, n. 60, e Massari, 1983, vol. I, pp. 111-113. Al v. 7 si è emendato "avran sofferto" in "avean sofferto" per analogia con il v. 4 ("avean patito").

37. Rinaldi, 1605, p. 1. La raccolta fu allestita da Giacomo Zoppino raccogliendo i testi già editi "in più volumi" e le "volanti rime" che circolavano ancora inedite. 
scorcio audace dei neologismi, il gioco vorticoso delle immagini analogiche rappresentano il correlativo di un'emozione altrimenti indefinibile, sono l'icona e la musica di particolari stati d'animo ben più che il racconto idealizzato di un'esistenza o, per dirla con il Rinaldi stesso, sono "creazione" ben più che "imitazione". 38

Fra le "volanti rime" per Barbara non incluse nelle Rime nuove e pubblicate poi da Giacomo Zoppino nel 1605 figurano cinque canzoni rivolte al Reni affinché ne dipinga il ritratto, nelle quali il Rinaldi sviluppa una sorta di colloquio fervido e riflessivo tra pittore e poeta: "Canta pingendo, i' pingerò cantando [...]". Tale serie fa parte di un gruppo di testi anteriori al 1603, poiché in essi Barbara risulta ancora in vita, e tuttavia esclusi dalle Rime nuove forse per il loro carattere troppo esplicito o nell'evocare un amore plebeo (Morta chioma, La donna s'appiglia al peggio, Fiato fetente) o sul piano teorico come manifesti di una nuova poetica (le Lodi della bellezza e le canzoni al Reni). Fin da subito il ritratto poetico di Barbara va ben oltre il canone descrittivo del petrarchismo e si dilata in un rapido avvicendarsi di quadretti analogici tra simbolo e mito per restituire l'effetto spaesante, impetuoso del bello. Così è nella meditazione filosofica delle Lodi della bellezza: "Ch'un monte è la bellezza, e 'l monte è un cielo, / ove un bosco di stelle alto frondeggia [...]"; 39 così è anche nella prima canzone al Reni:

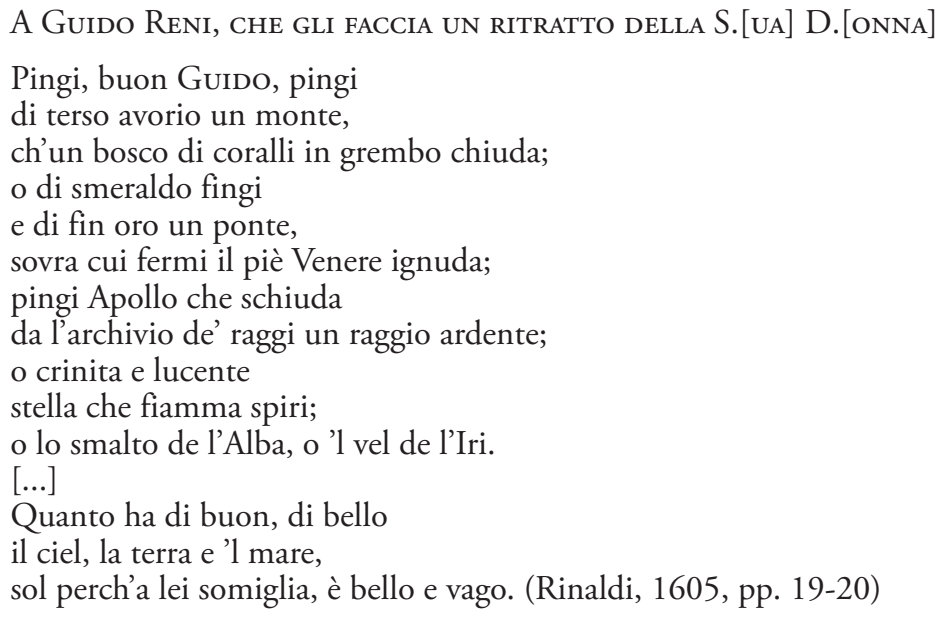

Le forme belle dell'intero universo non sono che riflessi luminosi di Barbara, come se lei fosse la luce stessa che illumina i sensi e accende l'immaginazione, e ciò permette al Rinaldi di riproporre ancora una volta il motivo dell'impossibilità di raffigurare lo splendore del sole:

Tante lucide forme e tanti onori, scherzi de la Natura,

38. Cfr. sopra la nota 43 e, per un'indagine tecnica dello stile del Rinaldi, Righi, 1980.

39. Cfr. Rinaldi, 1605, pp. 33, 3 e passim. 
son de la Diva mia faville e raggi;

son de l'Idolo mio forme e colori;

or chi ben s'assicura

pinger d'un aureo Sol gli aurei viaggi? (Rinaldi, 1605, p. 24)

Non si tratta più, però, di un modo per affermare il primato della poesia e anzi ora è il poeta a cedere il passo al pittore, più in grado di cogliere la luce inconfondibile e totalizzante di un volto amato:

Vorrei quell'aurea luce

ritrar, quel foco adorno,

lucido Sol ne le dipinte carte;

ma il poter manca e l'arte,

e quel ch'è gloria altrui, fora mio scorno.

Pingila tu felice,

o pittor de' pittori alba e fenice. (Rinaldi, 1605, p. 26)

E tuttavia anche il pittore, mentre traduce in forme e colori le "bellezze" e le "eccellenze" solo abbozzate dalla penna del poeta, rischia di produrre una catastrofe cosmica, un vortice di luce accecante e quasi un ritorno al caos primordiale. È una conclusione arguta e paradossale che intende esaltare l'effetto dinamico e impressivo dell'immagine come segreto comune delle arti sorelle:

Ohimè, pittor, che fai?

Mentre tu pingi le narrate forme,

non è più a sé conforme

il mondo, no, ch'ai fulminanti rai

del pinto volto fassi

fiamma il giel, polve il mar, cenere i sassi. (Rinaldi, 1605, p. 27)

Non sappiamo se il Reni abbia davvero realizzato quel ritratto o abbia anche solo attribuito i lineamenti di Barbara a qualche figura dei dipinti eseguiti per il Rinaldi. ${ }^{40}$ Certo è che di lì a poco, circondato dalla riprovazione tenace e accomodante del perbenismo bolognese, il Rinaldi sarebbe stato man mano condotto a riconoscere pubblicamente di avere oltrepassato un limite poetico e morale, pentendosi della superbia e della vanità della propria ricerca creativa. Già fra le rime inedite pubblicate dallo Zoppino nel 1605 figura qualche testo di generica ispirazione edificante contro le sregolatezze di "Amor lascivo",

40. Alcune espressioni del Rinaldi potrebbero al limite accreditare l'ipotesi che si tratti di un dipinto o un disegno realmente progettato o esistito: non solo formule come le "dipinte carte" (Rinaldi, 1605, p. 26) o il "pinto volto" (p. 27); ma anzitutto l'immagine del Tempo ormai sconfitto: "indarno la minaccia / quel gigante cursor, tromba di morte, / ché 'l tuo pennello, o Guido, al rio messaggio, / quasi radente acciar, tronca le braccia” (pp. 29-30) e "Canzon, Natura è vinta / e 'l Tempo invan rota i volanti giri, / se puote illustre fabro / far ch'ognor viva e spiri / il loquace color d'un pinto labro" (p. 30); e inoltre vi è la simultaneità di poesia e pittura: "Dunque tu col pennello et io col suono / farem costei famosa [...]" (p. 31). Molteplici sono anche le proposte di trasposizioni mitiche: ad esempio "Fingi prostrato il bel giudice d'Ida / che, rapito il bel pomo a Citerea, / per donarlo a costei curvi la fronte: / 'Ecco', le dica [...]” (p. 26). 
ma il documento più notevole è certo il sonetto premesso alla scelta di rime spirituali del Rinaldi inserite da Pietro Petracci nell'antologia delle Muse sacre del 1608. Qui il poeta è sul limite del silenzio definitivo, della rinuncia alla propria vocazione, e cerca tuttavia di piegare lo stile 'barbaro' delle Rime nuove a una poesia penitenziale sul tema del rifiuto dei diletti sensibili e della poesia stessa. Ed è anche l'ultima, indiretta menzione di Barbara con una sorta di amara, contrita nostalgia:

\author{
Al suo Padre Confessore \\ Mira il vasto ocean de' falli miei, \\ ondeggiante sepolcro a l'alma insana, \\ come tumideggiai di gloria vana, \\ come alzai d'orbo onor falsi trofei, \\ come l'onda fuggevole bevei \\ d'un lascivo piacer, come da tana \\ limosa tratta una bellezza umana, \\ strinsi in groppi di luce il Ciel con lei. \\ Or l'assenzio del duol l'acque amareggia \\ del pianto, e non più dentro al cieco ostello \\ d'Amor l'anima mia farneticheggia. \\ Donale tu pennuto arnese e bello, \\ fa' tu che per salir l'eterna Reggia, \\ se nel modo entrai verme, i' n'esca augello. (Petracci, 1608, p. 77)
}

Solo cinque anni erano bastati a trasformare il mito dolce e sfolgorante di Barbara in una povera "bellezza umana" uscita dalla "tana limosa" di una via malfamata. Per un poeta che aveva cercato di sottrarre la parola lirica alla tentazione del conformismo e dell'adattamento sociale, il silenzio sarebbe stato d'ora in poi il prezzo da pagare al disciplinamento crescente della cultura bolognese del primo Seicento dove proprio l'eterodossia creativa arginata e ridotta a regola morale sarà ciò che fa vivere la città e le dà prestigio. Ma l'affinità fra le arti sorelle, la parola come "musico pennel" che insieme muove l'immaginazione e interroga l'animo sarebbero rimaste comunque una scoperta e un'eredità cariche d'avvenire.

\title{
Riferimenti bibliografici
}

Bellori, G. P. (1672). Le vite de pittori, scultori et architetti moderni. Parte prima. Allillustriss. et eccellentiss. signore Gio. Battista Colbert [...]. In Roma: Per il Success. al Mascardi.

Bembo, P. (1997). Prose della volgar lingua, Gli Asolani, Rime (a cura di C. Dionisotti). Milano: TEA.

Besomi, O. (1969). Ricerche intorno alla "Lira" di G. B. Marino. Padova: Antenore.

Brusoni, G. (1647). Le Glorie de gli Incogniti o vero gli Huomini illustri dell'Accademia de'Signori Incogniti di Venetia. In Venetia: Appresso Francesco Valvasense stampator dell'Accademia. 
Campeggi, R. (1607). Filarmindo. Favola pastorale. (S.I.: s.n.). Ad istanza di Simone Parlasca.

Croce, B. (1928). Appunti di letteratura secentesca inedita o rara. II. Cesare Rinaldi, La Critica, 26, 61-68.

De Grazia, D. (1984). Le stampe dei Carracci con i disegni, le incisioni, le copie e i dipinti connessi. Catalogo critico (edizione italiana riveduta e aumentata tradotta e curata da A. Boschetto). Bologna: Alfa.

Di Benedetto, A. (1996). Con e intorno a Torquato Tasso. Napoli: Liguori.

Forni, G. (2018). "Il nemico interno". Politica, spiritualità e letteratura fra Cinque e Seicento. Novara: Interlinea.

Forni, G. (2019). Lirica e ironia nella poesia femminile del Rinascimento, Peloro, 4 (2), 53-87. doi: https://doi.org/10.6092/2499-8923/2019/4/2351

Fumaroli, M. (1995). La scuola del silenzio. Il senso delle immagini nel XVII secolo. Milano: Adelphi.

Gabinetto dei disegni e delle stampe della Pinacoteca nazionale di Bologna. (1975). Incisori bolognesi ed emiliani del '500. Appendice ai volumi "Incisori bolognesi ed emiliani del '600 e del '700" (schede di S. Ferrara, revisione di G. Gaeta Bertelà). Bologna: Associazione per le arti "Francesco Francia".

Giachino, L. (2003). "Amore è maggio che non corre a verno". Cinque saggi su lirici barocchi. Alessandria: Edizioni dell'Orso.

Groto, L. (1587). Delle rime. Nuovamente ristampate, \& ricorrette dal medesimo Autore. In Venetia: Appresso Fabio, \& Agostino Zoppini fratelli.

Guido Reni 1575-1642. (1988). Bologna: Nuova Alfa.

Malvasia, C. C. (1678). Felsina pittrice. Vite de’ pittori bolognesi. Alla Maestà Christianissima di Luigi XIIII re di Francia e di Navarra il sempre vittorioso [...], Divisa in duoi Tomi; con Indici in fine copiosissimi [...]. In Bologna: Per l'Erede di Domenico Barbieri, Ad instanza di Gio. Francesco Davico, detto il Turrino.

Marziale, M. V. (2007). Epigrammi (a cura di S. Beta). Milano: Arnoldo Mondadori.

Massari, S. (1983). Giulio Bonasone (2 Voll.). Roma: Quasar.

Pancaldi, G. P. (1636). Allillustriss. sig. marchese Virgilio Malvezzi per la morte del signor Cesare Rinaldi. In Bologna: Presso Giacomo Monti, e Carlo Zenero.

Pepper, S. (1983). "Bacchus and Ariadne" in the Los Angeles County Museum: The "Scherzo" as Artistic Mode, The Burlington Magazine, 125 (959), 68-75.

Perini, G. (1995). "Ut pictura poesis": l'Accademia dei Gelati e le arti figurative. In D. S. Chambers \& F. Quiviger (Edd.), Italian Academies of the Sixteenth Century (pp. 113-126). London: The Warburg Institute-University of London.

Petracci P. (Ed.). (1608). Le Muse Sacre. Scelta di Rime Spirituali, de’ più Eccellenti Autori d'Italia. In Venetia: Appresso Evangelista Deuchino e Gio. Batt. Pulciano.

Piantoni, L. (2013). "Lasciva e penitente”. Nuovi sondaggi sul tema della Maddalena nella poesia religiosa del Seicento. Studi secenteschi, 54, 25-48.

Pich, F. (2007). "Né in ciò me sol, ma l'arte insieme accuso". I sonetti a Tiziano nella tradizione delle rime per ritratto. In S. Carrai (Ed.), Giovanni Della Casa ecclesiastico e scrittore. Atti del convegno: Firenze-Borgo San Lorenzo, 20-22 novembre 2003 (pp. 401-444). Roma: Edizioni di Storia e Letteratura.

Pigna, G. B., Calcagnini, C., \& Ariosto, L. (1553). Carmina. Venetiis: ex Officina Erasmiana Vincentii Valgrisii.

Raimondi, E. (1995). Il colore eloquente. Letteratura e arte barocca. Bologna: il Mulino. Righi, D. (1980). L'indice metaforico di uno scrittore del Seicento, Lingua e stile, 15 (2), 211-232. 
Rinaldi, C. (1588). De’ Madrigali Prima, \& Seconda Parte. Al Molto Illust. Sig. il Sig. Conte Alberto Castelli [...]. In Bologna: per Alessandro Benacci.

Rinaldi, C. (1590). Delle rime parte terza. Alla ill.ma et ecc.ma sig.ra la sig.ra Pellegrina Capello Bentivoglia. In Bologna: Per Vittorio Benacci.

Rinaldi, C. (1591). Delle rime parte quarta. Con la scielta dell'altre tre parti. In Bologna: Per Vittorio Benacci.

Rinaldi, C. (1598). Delle rime parte sesta. Al Sereniss. Sig. Don Cesare d'Este Duca di Modona. In Bologna: Per gli Her. di Gio. Rossi.

Rinaldi, C. (1601). Canzoniere. All'Ill.mo et Reverendiss.mo Mons.r Giuliano della Rovere. In Bologna: Per gli Her. di Gio. Rossi.

Rinaldi, C. (1603). Rime nuove. All'illustriss. et eccellentiss. sig. il signor D. Ferrante Gonzaga Prencipe di Molfetta, Signor di Guastalla, etc. In Bologna: Presso gli Heredi di Giovanni Rossi.

Rinaldi, C. (1605). Rime. All'Illustriss. \& Eccellentiss. Prencipe Il Sig. D. Virginio Orsini Duca di Bracciano, Generale di S. Chiesa. In Venetia: A Sant'Anzolo.

Rinaldi, C. (1617). Lettere. Allillustrissimo, et reverendiss. sig. il signor Cardinal d'Este. In Venetia: Appresso Tomaso Baglioni.

Rinaldi, C. (1619). Rime. In questa terza impressione dal medesimo autore riviste, $e$ ricorrette [...]. In Bologna: per Gieronimo Mascheroni.

Rinaldi, C. (1620). Delle lettere volume secondo. Al Sereniss. Ferdinando Gonzaga Duca di Mantova, e di Monferrato. In Bologna: ad istanza di Pelegrino Golfarini.

Ritrovato, S. (2005). "Per te non di te canto". I madrigali di Cesare Rinaldi. Manziana: Vecchiarelli.

Ruiz Sánchez, M. (1998). Las metamorfosis del mármol. Mito y obra de arte en los epigramas neolatinos. Minerva. 88, 12, 161-179. Disponibile su https://revistas. uva.es/index.php/minerva/article/view/2978. 


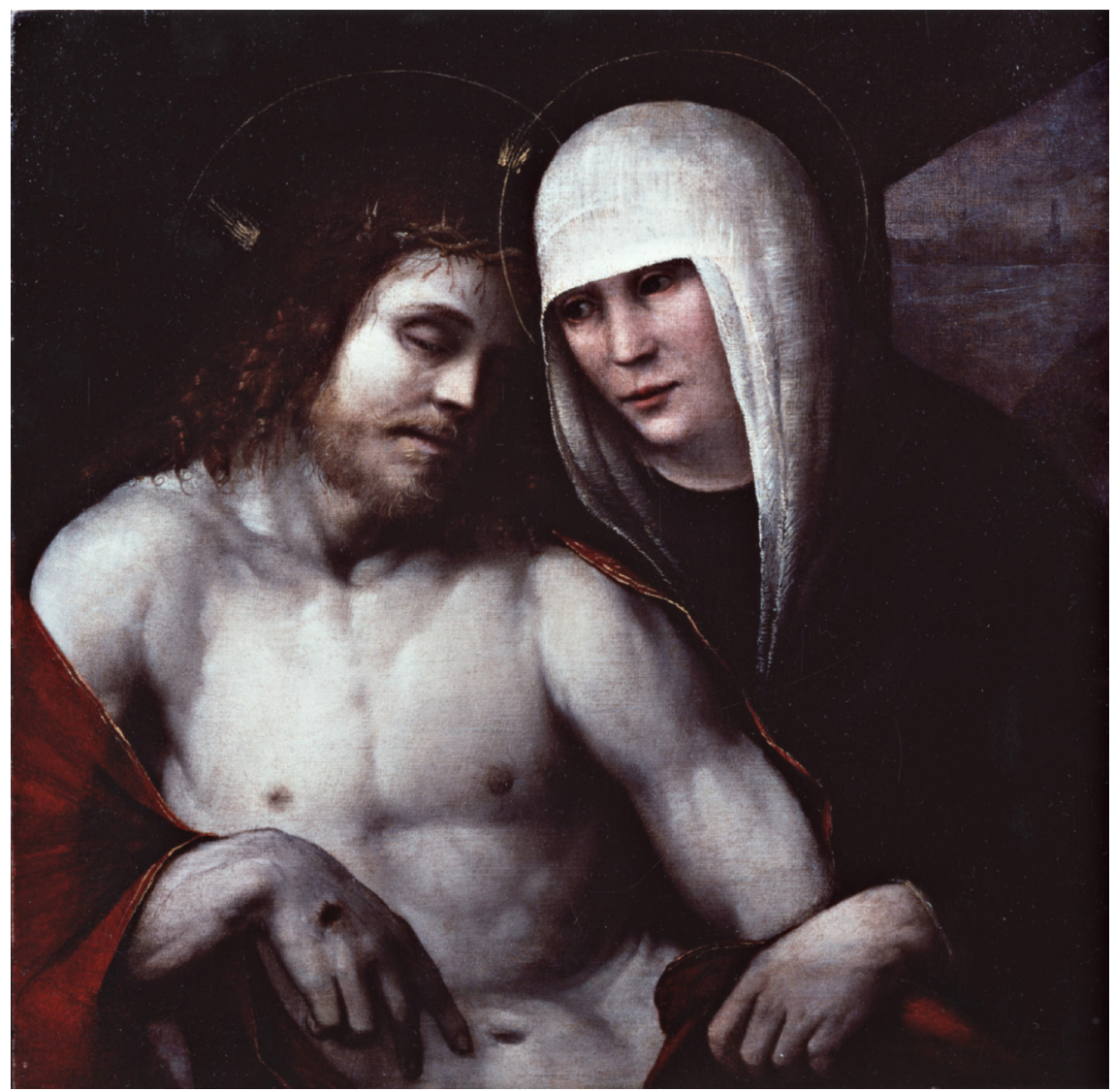

Dosso Dossi, Pietà, prima metà del XVI sec. (Fototeca Federico Zeri, Bologna) 


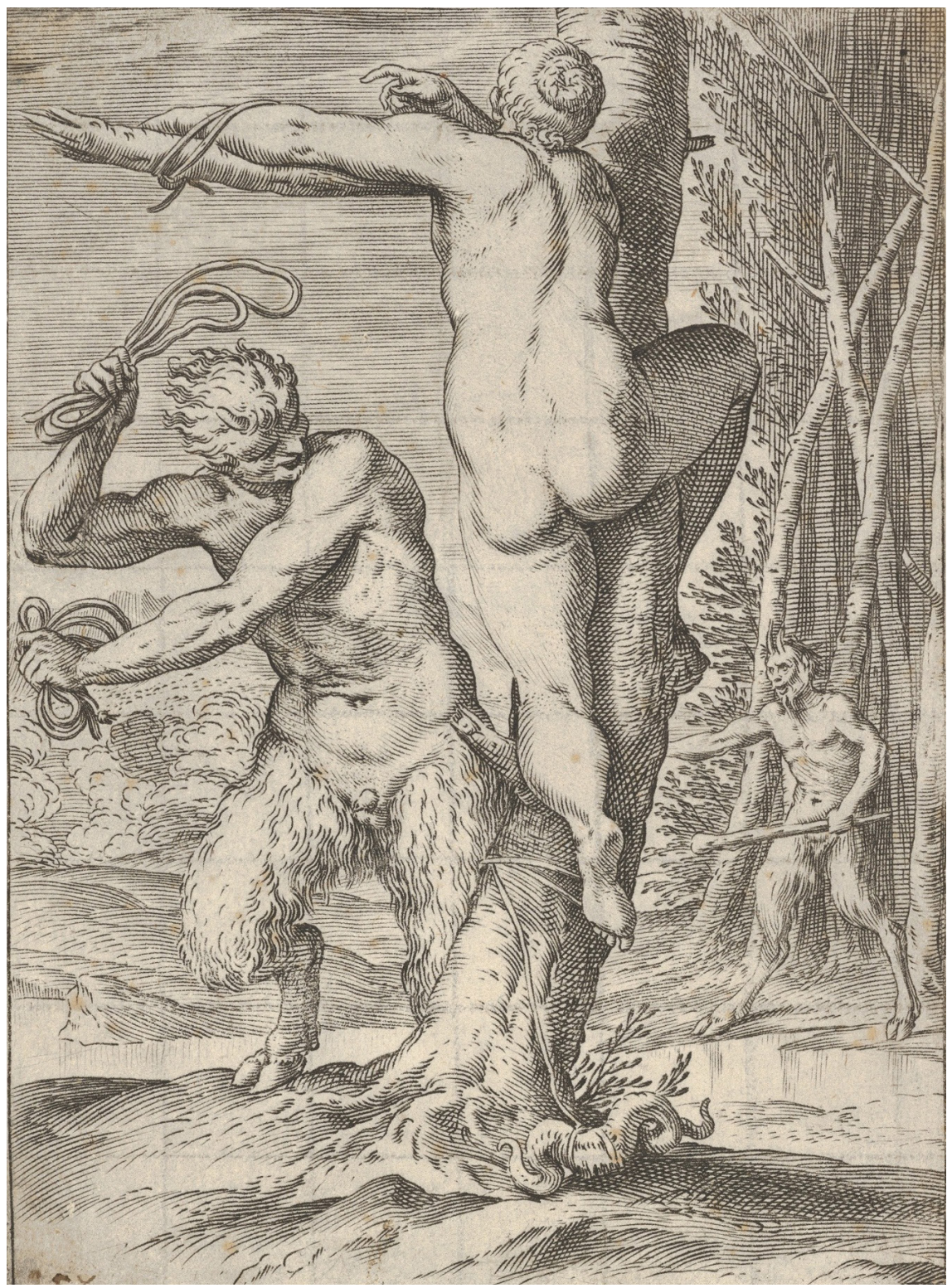

Agostino Carracci, Le Lascivie. Satiro che batte una ninfa, 1584-1595 


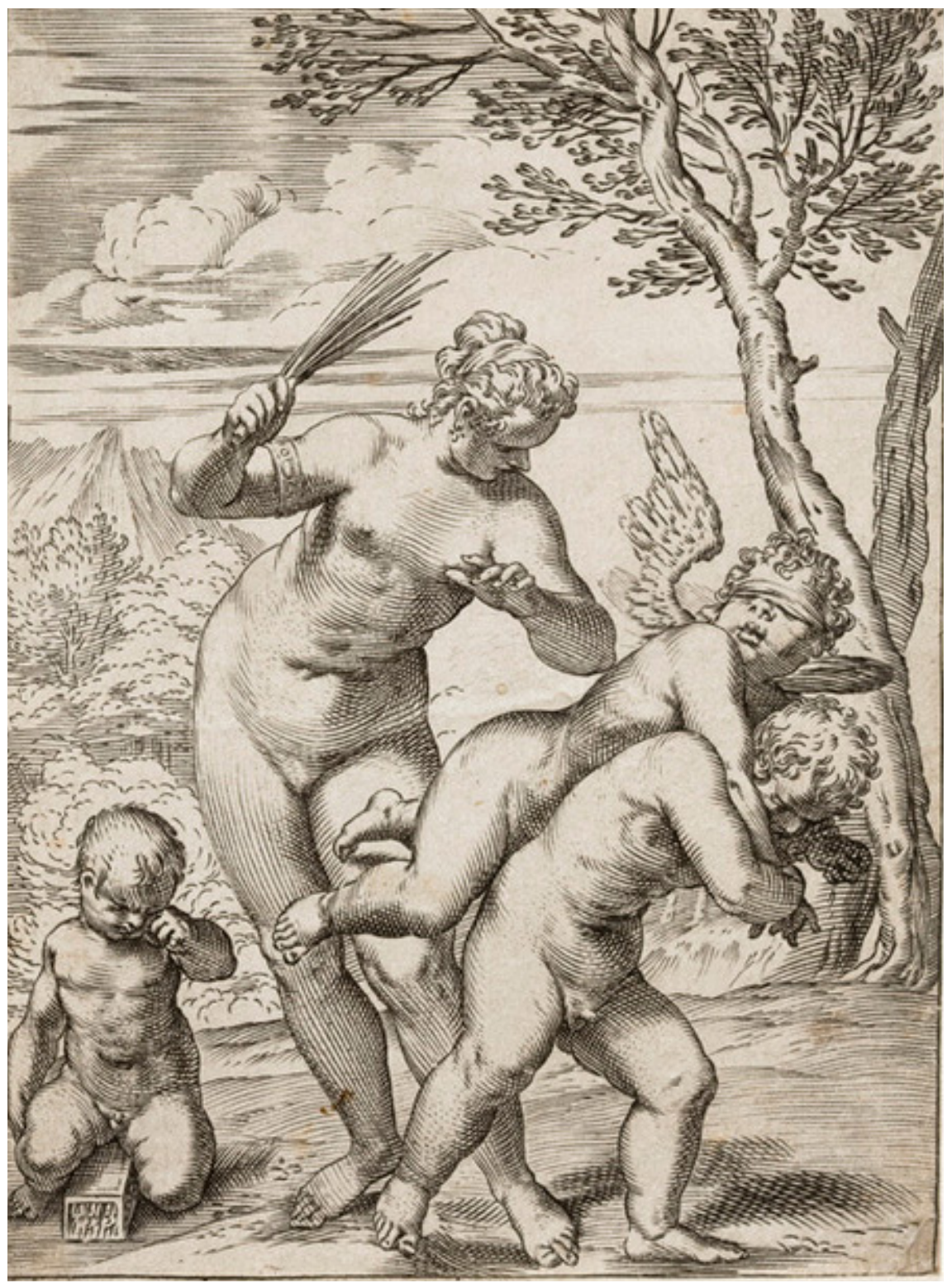

Agostino Carracci, Le Lascivie. Venere punisce l'Amore profano, 1584-1595. 